\title{
In Vitro-In Vivo Correlation: Shades on Some Non-Conventional Dosage Forms
}

\author{
Abdulwahab Barakat ${ }^{1,2}$, Johannes Krämer ${ }^{2,}$, Cristiane de Souza Carvalho ${ }^{3}$, and Claus-Michael \\ Lehr $^{1,3}$ \\ ${ }^{1}$ Department of Biopharmaceutics and Pharmaceutical Technology, Saarland University, Saarbrücken, Germany \\ 2 PHAST Development GmbH \& Co. KG, Constance, Germany \\ ${ }^{3}$ Helmholtz-Institute for Pharmaceutical Research Saarland (HIPS), Saarland University, Saarbrücken, Germany
}

\begin{abstract}
In vitro-in vivo correlation (IVIVC) is an important biopharmaceutical tool that is essential during the pharmaceutical development, quality control, and pre- and post-approval changes. IVIVC has been well established for conventional solid oral dosage forms whereby in vitro drug release profiles are usually correlated to in vivo pharmacokinetic parameters. However, there is poor guidance regarding other non-conventional dosage forms. For medicated chewing gums, drug release methodologies were developed, and the results thereof were compared with in vivo chewout studies showing good correlation. Since medicated gums are directly accessible, IVIVC is based on true release data with no interference by absorption phenomena as in the case of orals. Orally inhaled products are different, and IVIVC is challenging. No standardized in vitro drug release testing method exists for testing inhaled products. At this point, aerodynamic particle size measurement (APSD) is the only quality attribute linked to the in vivo performance of the drug product. Alternatively, computational fluid dynamics for the prediction of aerosol deposition within the respiratory tract is used.
\end{abstract}

KEYWORDS: Medicated gums; inhalation; dissolution; IVIVC; particle size.

\section{INTRODUCTION}

$\mathrm{n}$ vitro-in vivo correlation (IVIVC) plays a major role in the pharmaceutical development of dosage forms. In recent years, the concept and application of IVIVC for pharmaceutical dosage forms have been a main focus of the pharmaceutical industry, academic research, and regulatory sectors. IVIVC's main objective is to link the surrogate parameter of the therapeutic efficacy (i.e., bioavailability, $\mathrm{BA})$ to the in vitro drug release kinetics. The primary goal is to support biowaivers and hence reduce the number of human BA studies during development of new drug products (1). IVIVCs are the prevalent way to establish meaningful dissolution specifications in quality control testing to ascertain that all batches released are bioequivalent to the model for which safety and efficacy are proven. In lifecycle management, IVIVC may support scale-up and post-approval changes. IVIVC is the link between in vivo and in vitro performance testing of a drug product, with relevance for both drug product development and quality control (2).

\section{DEFINITIONS}

The USP and the FDA define IVIVC differently from the definition above.

\section{USP Definition of IVIVC}

"The establishment of a rational relationship between a biological property, or a parameter derived from a biological property produced by a dosage form, and a physicochemical property or characteristic of the same dosage form (3)."

\section{FDA Definition of IVIVC}

"A predictive mathematical model describing the relationship between an in vitro property of a dosage form and an in vivo response (4)."

The in vitro characteristic is the rate of drug release, while the in vivo response is the plasma drug concentration-time profiles of the drug or the active moiety. The main purpose of IVIVC is to describe a functional relationship to allow the prediction of blood drug concentration profiles on the basis of in vitro drug release kinetics. USP General Chapter $<1088>$ provides guidance for both in vitro and in vivo testing of drugs. The prerequisite for a successful IVIVC is that in vivo drug release rather than absorption is the rate-limiting step. The Biopharmaceutics Classification System (BCS) expresses the kinetic in vivo processes dissolution and absorption by the in vitro solubility and even in vitro permeability of the drug substance. It is therefore limited to immediaterelease (IR) oral drug products. The BCS provides a rationale for estimating the likelihood of an IVIVC for a certain IR product, which is depicted below (5):

\footnotetext{
*Corresponding author.
} 


\begin{tabular}{|c|c|c|l|}
\hline \multicolumn{4}{|c|}{$\begin{array}{c}\text { In Vitro-In Vivo (IVIVC) Correlation Expectations for Immediate-Release } \\
\text { Products Based on Biopharmaceutics Classification System }\end{array}$} \\
\hline Class & Solubility & Permeability & \multicolumn{1}{c|}{ IVIV Correlation Expectation } \\
\hline I & High & High & $\begin{array}{l}\text { IVIV correlation if dissolution rate is slower than gastric emptying } \\
\text { rate. Otherwise, limited or no correlation is expected. }\end{array}$ \\
\hline II & Low & High & $\begin{array}{l}\text { IVIV correlation expected if in vitro dissolution rate is similar to } \\
\text { in vivo dissolution rate unless dose is very high. }\end{array}$ \\
\hline III & High & Low & $\begin{array}{l}\text { Absorption (permeability) is rate determining and limited, or no } \\
\text { IVIV correlation with dissolution rate. }\end{array}$ \\
\hline IV & Low & Low & Limited or no IVIV correlation expected. \\
\hline
\end{tabular}

\section{CORRELATION LEVELS OF IVIVC}

Three levels have been defined by the FDA guidance (6). The concept of correlation level is based upon the ability of the correlation to reflect the complete plasma drug level-time profile that will result from administration of a given form.

\section{Level A Correlation}

This level is the highest category of correlation and represents a point-to-point relationship between in vitro dissolution rate and in vivo input rate of the drug dosage form. It is the preferred correlation level to be achieved. Generally, the percentage of drug absorbed may be calculated by means of model-dependent techniques such as the Wagner-Nelson or Loo-Riegelman methods or by model-independent numerical deconvolution. These techniques represent a major advance over the singlepoint approach in that they utilize all of the dissolution and plasma level data available in developing the correlation. The Level A correlation is reversible.

\section{Level B Correlation}

This level utilizes the principles of statistical moment analysis. In this level, the mean in vitro dissolution time $\left(M D T_{\text {vitro }}\right)$ of the product is compared with either the mean in vivo residence time (MRT) or the mean in vivo dissolution time $\left(M D T_{\text {vivo }}\right)$. As data reduction is performed, at least three batches need to be investigated. Level $B$ correlation is not reversible.

\section{Level C Correlation}

In this level of correlation, one dissolution time point (e.g., $t_{50 \%}, t_{90 \%}$ ) is compared with one mean pharmacokinetic parameter such as area under the curve $(A \cup C)$ or $C_{\max }$. This is the weakest level of correlation. A Level $C$ correlation can be useful in the early stages of formulation development when pilot formulations are being selected. A multiple Level C correlation relates more than one pharmacokinetic parameter to corresponding coordinates of the dissolution profiles. As for a Level B correlation, three or more batches need to be investigated, and the correlation is not reversible.

\section{LIMITATIONS TO IVIVC}

- More than one variation of an oral drug product is needed plus the oral solution in the case of numerical deconvolution.

- Pharmacokinetics of the drug should be "linear" within the dosage range. Pharmacokinetic processes need to be invariant over time.

- Absorption must not be the limiting factor, which means that drug release must be the slower phenomenon compared with absorption. However, it should be considered that drug release and dissolution may not be identical under in vivo conditions.

\section{SPECIAL DOSAGE FORMS}

The principles of IVIVC model development have been successfully applied to oral dosage forms. However, the rules for developing and validating IVIVC models for novel and non-oral dosage forms/delivery systems (microspheres, implants, liposomes, gums, etc.) are still subject to future standardization. The process for establishing IVIVC for medicated gums is used as an example to prove the feasibility of correlation with a slightly different approach to access meaningful in vivo parameters. In the case of orally inhaled products, additional research on both the in vivo and the in vivo side is needed.

\section{Medicated Gums}

Medicated chewing gums are solid oral dosage forms intended to be activated by the patient by chewing. They provide controlled release of the active as a function of mastication. Drug release stops when the dosage form is swallowed. Drug release tests for medicated chewing gums have been developed using compendial apparatus. As for conventional oral products, the compendial apparatus allow, among other things, the determination of the influence of composition and the manufacturing process of the gums on drug release $(7,8)$.

No classical IVIVC procedure has been reported for medicated chewing gums. Pharmacokinetic data from several chewing gums have shown high variability in terms of plasma concentration-time curves. This is due to the complexity of this route of administration. When the gum is chewed, part of the API is absorbed in the buccal cavity, whereas the rest will be swallowed and absorbed 
distally in the gastrointestinal tract where it might undergo first-pass metabolism. Taking this high variability into consideration, a proper correlation of in vitro release data to pharmacokinetic data may not be developed. Unlike conventional oral dosage forms, medicated chewing gums provide access to the dosage form at the site of drug release after administration. By determining the residual content, drug release can be quantified precisely. Thus, studies correlating in vitro dissolution data from compendial apparatus to in vivo chew-out study data were published $(9,10)$ the mode and the mechanism of release and the site of application differ significantly from other conventional solid oral dosage forms and require a special consideration to extract meaningful information from clinical studies. In the current study, suitable drug release methodology was developed to predict the in vivo performance of an investigated chewing gum product. Different parameters of the drug release testing apparatus described in the $\mathrm{Ph}$. Eur. and Pharmeuropa were evaluated. Drug release data indicate that the parameters, chewing distance, chewing frequency and twisting motion, affect the drug release. Higher drug release was observed when the frequency was changed from 40 chews $/ \mathrm{min}$ to 60 chews $/ \mathrm{min}$ for apparatus $A$ and $B$, as was the case for the twisting motion when changed from 20lu00ba to 40lu00ba for apparatus B. As far as the chewing distance is concerned, the release rate was in the following order; apparatus A: $0.3 \mathrm{~mm}>0.5$ $\mathrm{mm}>0.7 \mathrm{~mm}$; apparatus $\mathrm{B}: 1.4 \mathrm{~mm}>1.6 \mathrm{~mm}>1.8 \mathrm{~mm}$. A suitable apparatus set-up for in vitro release testing was identified. The method will be useful for the establishment of in vitro in vivo correlations (IVIVC. The drug remaining in the chewed gum allows precise determination of the amount released and hence the performance in vivo (11).

\section{Orally Inhaled Drug Products (OIDPs)}

As described above, IVIVC is established by correlating in vitro drug release to in vivo pharmacokinetic data. However, because of the absence of a standardized in vitro drug release test method for OIDPs of the dry powder inhaler type, classical IVIVC is not yet possible.

It is assumed that cascade impactor deposition data may correlate to the drug deposition in lungs (12). Due to the complexity of this route of application, a comparison of aerodynamic particle size distribution (APSD) profiles may not confirm bioequivalence. Many factors differ from one patient to another including inhalation maneuver and the anatomy, physiology, and disease state of patient airways. This suggests that depending on the drug and disease to be treated, different APSDs could occur (13). In vitro physical airway models and in silico computer models have been studied to achieve IVIVC (14). A product was approved in Europe after intensive pharmacokinetic investigation and drug deposition IVIVC modeling (15).

In vitro-ex vivo correlation has been reported. BeckBroichsitter et al. (16) developed a controlled delivery, liposome-based formulation and accessed the in vitro release. They used an isolated, perfused, and ventilated lung model to measure pulmonary absorption. Very good agreement was achieved between the in vitro and ex vivo results based on a single-point correlation.

\section{IN VITRO PERFORMANCE TESTING}

Drug release testing of orally inhaled products is an ongoing research topic. However, its relevance to the biological situation is a matter of discussion $(17,18)$. The USP Inhalation Ad Hoc Advisory Panel "could not find compelling evidence suggesting that such dissolution testing is kinetically and/or clinically crucial for currently approved inhalation drug products (19)." Some proposals about dissolution testing of inhalation products using the paddle apparatus, flow-through cell, Franz diffusion cell apparatus, and Transwell diffusion apparatus were published (20-23).

\section{PULMONARY BIOPHARMACEUTICAL CLASSIFICATION SYSTEM (pBCS)}

An important publication by Eixarch et al. (24) proposed a $\mathrm{pBCS}$ as an extension to the well-known BSC. The proposed system takes into consideration the biological properties of the lung along with formulation and physicochemical properties of the drug. Biological properties include metabolism, drug-drug interactions, efflux transporters, protein binding, clearance, and mucus and surfactant. In addition to solubility and permeability, physicochemical properties include aerodynamic characteristics that will affect particle deposition. To establish the system, biorelevant solubility data and, most importantly, permeability will be needed. To access the permeability through the air-blood barriers in the lung, many in vitro models were developed based on cell culture and recently tissue engineering approaches (25).

\section{Locally Acting OIDPs}

In the case of locally acting drug products with no absorption, the classical correlation parameter derived from blood level analyses is not available. For this special case, alternative biological properties are highly desirable. Ongoing research may provide a model for biorelevant testing of pulmonary OIDPs, which may allow excursion from systemically to locally acting drug based on a modified BCS taking into account the permeability of pulmonary barriers (26).

\section{REFERENCES}

1. Lu, Y.; Kim, S.; Park, K. In vitro-in vivo correlation: Perspectives on model development.Int.J.Pharm. 2011, 418 (1), 142-148. DOI: 10.1016/j.ijpharm.2011.01.010.

2. Emami, J. In vitro-In vivo Correlation: From Theory to Applications. J. Pharm. Pharmaceut. Sci. 2006, 9 (2), 169-189.

3. <1088> In Vitro and In Vivo Evaluation of Dosage Forms. In The United States Pharmacopeia and 
National Formulary USP 37-NF 32; The United States Pharmacopeial Convention, Inc.: Rockville, MD, 2014.

4. Extended Release Oral Dosage Forms: Development, Evaluation, and Application of In Vitro/In Vivo Correlations; Guidance for Industry; U.S. Department of Health and Human Services, Food and Drug Administration, Center for Drug Evaluation and Research (CDER), U.S. Government Printing Office: Washington, DC, 1997.

5. Bermejo, M. Dissolution Methodologies and IVIVC. Presented at Strategies for Oral Drug Delivery, Lake Tahoe, NV, March 10-16, 2002.

6. Cardot, J.-M.; Beyssac, E.; Alric, M. In Vitro-In Vivo Corellation:Importance of Dissolution in IVIVC. Dissolution Technol. 2007, 14 (1), 15-19. DOI: 10.14227/ DT140107P15.

7. Gajendran, J.; Kraemer, J.; Knudsen, S. R. Product Performance Test for Medicated Chewing Gums. Pharm. Forum 2008, 34 (3), 843-847.

8. Morjaria, Y.; Irwin, W. J.; Barnett, P.X.; Chan, R. S.; Conway, B. R. In Vitro Release of Nicotine From Chewing Gum Formulations. Dissolution Technol. 2004, 11 (2), 12-15. DOI: 10.14227/DT110204P12.

9. Gajendran, J.; Kraemer, J.; Langguth, P. In vivo predictive release methods for medicated chewing gums. Biopharm. Drug Dispos. 2012, 33 (7), 417-424. DOI: 10.1002/bdd.1796.

10. Kvist, L. C.; Andersson, S.-B.; Berglund, J.; Wennergren, B.; Fors, S. M. Equipment for drug release testing of medicated chewing gums.J. Pharm. Biomed.Anal. 2000, 22 (3), 405-411. DOI: 10.1016/S0731-7085(99)00307-6.

11. Woodford, D. W.; Lesko, L. J. Relative bioavailability of aspirin gum. J. Pharm. Sci. 1981, 70 (12), 1341-1343. DOI: $10.1002 / j p s .2600701213$.

12. Mitchell, J.; Newman, S.; Chan, H.-K. In Vitro and In Vivo Aspects of Cascade Impactor Tests and Inhaler Performance: A Review. AAPS PharmSciTech 2007, 8 (4), 237-248. DOI: 10.1208/pt0804110.

13. Evans, C.; Cipolla, D.; Chesworth, T.; Agurell, E.; Ahrens, R.; Conner, D.; Dissanayake, S.; Dolovich, M.; Doub, W.; Fuglsang, A.; García Arieta, A.; Golden, M.; Hermann, R.; Hochhaus, G.; Holmes, S.; Lafferty, P.; Lyapustina, S.; Nair, P.; O'Connor, D.; Parkins, D.; Peterson, I.; Reisner, C.; Sandell, D.; Singh, G. J. P.; Weda, M.; Watson, P. Equivalence Considerations for Orally Inhaled Products for Local Action-ISAM/IPAC-RS European Workshop Report. J. Aerosol Med. Pulm. Drug Delivery 2012, 25 (3), 117-139. DOI:10.1089/jamp.2011.0968.

14. Byron, P. R.; Hindle, M.; Lange, C. F.; Longest, P. W.; McRobbie, D.; Oldham, M. J.; Olsson, B.; Thiel, C. G.; Wachtel, H.; Finlay, W. H. In Vivo-In Vitro Correlations: Predicting Pulmonary Drug Deposition from Pharmaceutical Aerosols. J. Aerosol Med. Pulm. Drug Delivery 2010, 23 (S2), S59-S69.

15. Lähelmä, S.; Sairanen, U.; Haikarainen, J.; Korhonen, J.; Vahteristo, M.; Fuhr, R.; Kirjavainen, M. Equivalent Lung Dose and Systemic Exposure of Budesonide/
Formoterol Combination via Easyhaler and Turbuhaler. J. Aerosol Med. Pulm. Drug Delivery 2015, 28 (0), 1-12. DOI: 10.1089/jamp.2014.1195.

16. Beck-Broichsitter, M.; Rieger, M.; Reul, R.; Gessler, T.; Seeger, W.; Schmehl, T. Correlation of drug release with pulmonary drug absorption profiles for nebulizable liposomal formulations. Eur. J. Pharm. Biopharm. 2013, 84 (1), 106-114. DOI: 10.1016/j.ejpb.2012.12.003.

17. Riley, T.; Christopher, D.; Arp, J.; Casazza, A.; Colombani, A.; Cooper, A.; Dey, M.; Maas, J.; Mitchell, J.; Reiners, M.; Sigari, N.; Tougas, T.; Lyapustina, S. Challenges with Developing In Vitro Dissolution Tests for Orally Inhaled Products (OIPs). AAPS PharmSciTech 2012, 13 (3), 978989.

18. Kanapilly, G. M.; Raabe, O. G.; Goh, C. H.; Chimenti, R. A. Measurement of in Vitro Dissolution of Aerosol Particles for Comparison to in Vivo Dissolution in the Lower Respiratory Tract after Inhalation. Health Phys. 1973, 24 (5), 497-507. DOI: 10.1097/00004032$197305000-00004$.

19. Gray, V. A.; Hickey, A. J.; Balmer, P.; Davies, N. M.; Dunbar, C.; Foster, T. S.; Olsson, B. L.; Sakagami, M.; Shah, V. P.; Smurthwaite, M. J. The Inhalation Ad Hoc Advisory Panel for the USP Performance Tests of Inhalation Dosage Forms. Pharm. Forum 2008, 34 (4), 1068-1074.

20. May, S.; Jensen, B.; Wolkenhauer, M.; Schneider, M.; Lehr, C. M. Dissolution Techniques for In Vitro Testing of Dry Powders for Inhalation. Pharm. Res. 2012, 29 (8), 2157-2166. DOI: 10.1007/s11095-012-0744-2.

21. Arora, D.; Shah, K. A.; Halquist, M. S.; Sakagami, M. In Vitro Aqueous Fluid-Capacity-Limited Dissolution Testing of Respirable Aerosol Drug Particles Generated from Inhaler Products. Pharm. Res. 2010, 27 (5), 786795. DOI: 10.1007/s11095-010-0070-5.

22. Son, Y.-J.; McConville, J. T. Development of a standardized dissolution test method for inhaled pharmaceutical formulations. Int. J. Pharm. 2009, 382 (1-2), 15-22. DOI: 10.1016/j.ijpharm.2009.07.034.

23. Davies, N. M.; Feddah, M. R. A novel method for assessing dissolution of aerosol inhaler products. Int. J. Pharm. 2003, 255 (1-2), 175-187. DOI: 10.1016/S03785173(03)00091-7.

24. Eixarch, H.; Haltner-Ukomadu, E.; Beisswenger, C.; Bock, U. Drug Delivery to the Lung: Permeability and Physicochemical Characteristics of Drugs as the Basis for a Pulmonary Biopharmaceutical Classification System (pBCS ). J. Epithelial Biol. Pharmacol. 2010, 3, $1-14$.

25. de Souza Carvalho, C.; Daum, N.; Lehr, C.-M. Carrier interactions with the biological barriers of the lung: Advanced in vitro models and challenges for pulmonary drug delivery. Adv. Drug Deliv. Rev. 2014, 75, 129-140. DOI: 10.1016/j.addr.2014.05.014.

26. Schanker, L. S. Drug absorption from the lung. Biochem. Pharmacol. 1978, 27 (4), 381-385. DOI: 10.1016/00062952(78)90365-9. 